\title{
Efeitos da vibração do corpo todo sobre a força e potência muscular de idosos: Uma revisão sistemática
}

\author{
Effects of whole-body vibration on muscle strength and power of elderly: \\ A systematic review
}

Monique Opuszcka Campos, ${ }^{1}$ Paulo Sergio Chagas Gomes ${ }^{1 *}$

ARTIGO DE REVISÃO | REVIEW ARTICLE

\begin{abstract}
RESUMO
O objetivo desta revisão sistemática foi sintetizar as evidências científicas disponíveis sobre a utilização do treino com vibração do corpo todo (VCT) como uma alternativa para a obtenção de modificações efetivas sobre a força e a potência muscular de idosos. Foram recuperados artigos das bases de dados Medline, SciELO, Lilacs, Biblioteca Cochrane, PEDro e Science Citation Index. A avaliação da qualidade dos estudos foi realizada pela escala PEDro e a análise dos estudos foi feita por meio de uma revisão crítica dos conteúdos. Dos 91 estudos recuperados, 75 foram excluídos e 16 atenderam aos critérios de seleção. A maioria (68.8\%) apresentou moderada a alta qualidade metodológica. A VCT associada aos exercícios isométricos e dinâmicos demonstrou-se como uma alternativa de intervenção terapêutica para a melhoria da força muscular de idosos. No entanto, as características dos delineamentos experimentais e a presença de ameaças à validade interna (ausência da condição controle para o estímulo vibratório) dificultam estabelecer os efeitos adicionais da VCT na população alvo. Achados divergentes foram encontrados para o efeito da VCT sobre a potência muscular. São necessários ensaios clínicos randomizados e controlados para estabelecer a real efetividade deste tipo de intervenção.

Palavras-chave: envelhecimento, estímulo vibratório, exercício físico, sarcopenia
\end{abstract}

ABSTRACT

The aim of this systematic review was to summarize available scientific evidence on the utilization of whole body vibration as an alternative method to promote effective modifications on muscle strength and power in the aging population. Scientific studies were retrieved from the following databases: Medline, Scielo, Lillacs, Cochrane Library, PEDro and Science Citation Index. The PEDro scale was used to assess the quality of the included studies, while content went through a critical analysis. From the 91 studies retrieved, 75 were excluded and 16 attended the selection criteria. From the16, the majority $(68.8 \%)$ presented from moderate to high methodological quality. Whole-body vibration associated to both isometric and dynamic exercises seemed to constitute an alternative for therapeutic intervention to improve muscular strength and power of healthy elderly. However, due to the characteristics of the designs of the studies reviewed and the threats to their internal validity (i.e., the absence of the control condition to the vibratory stimulus) it was challenging to establish the additional effects of the whole-body vibration on the target population. Divergent findings were found for the whole-body vibration effect on muscular power. It is still necessary to conduct randomized control trials to establish the real effectiveness of this kind of intervention.

Keywords: aging, vibratory stimulus, physical exercise, sarcopenia

Artigo recebido a 25.02.2013; $1^{\text {a }}$ Revisão 12.06.2013; Aceite 29.07.2013

${ }^{1}$ Programa de Pós-graduação em Ciências do Exercício e do Esporte da Universidade Gama Filho, Rio de Janeiro - RJ, Brasil

* Autor correspondente: Laboratório Crossbridges, Programa de Pós-graduação em Ciências do Exercício e do Esporte, Universidade Gama Filho, Rua Manoel Vitorino 553, Piedade, CEP: 20748-900 Rio de Janeiro, RJ, Brasil; E-mail: gomespscg@yahoo.com.br 


\section{INTRODUÇÃO}

O envelhecimento está associado à deterioração dos sistemas neuromuscular (Frontera et al., 2000) e sensorial (Evans et al., 2002), bem como a do processamento central (Fathi et al., 2010), fatores estes que estão diretamente envolvidos com comprometimentos físicos, prejuízos psicológicos e consequente perda da autonomia e qualidade de vida (Chu, Chiu, \& Chi, 2006).

A redução da massa e da força muscular representa uma grande preocupação diante do envelhecimento, uma vez que contribuem para o aumento do risco de quedas (Chu et al., 2006), fraturas (Baumgartner et al., 1998), perda da independência funcional e apresentam alto impacto na qualidade de vida (Chu et al., 2006).

O declínio da massa muscular, e consequentemente da força, está associado ao aumento da sarcopenia, cujos mecanismos desencadeadores incluem: a diminuição no número e no tamanho das fibras musculares (Frontera et al., 2000), a redução na síntese proteica (Rooyackers, Adey, Ades, \& Nair, 1996), o aumento do componente não contrátil do tecido muscular (Kent-Braun, Ng, \& Young, 2000), a diminuição do número e no recrutamento das unidades motoras (Erim, Beg, Burke, \& de Luca, 1999), as alterações na arquitetura muscular e nas propriedades da estrutura tendínea (Onambele, Narici, \& Maganaris, 2006), assim como a redução da função mitocondrial (Short et al., 2005).

A presença da sarcopenia pode, adicionalmente, contribuir para o desenvolvimento de desordens crónicas comummente relacionadas ao envelhecimento (Di Monaco, Vallero, Di Monaco, \& Tappero, 2010; Miljkovic-Gacic et al., 2008), ressaltando ainda mais a necessidade de valorização de programas de intervenção voltados para a otimização do desempenho muscular nesta população.

O exercício físico é capaz de induzir adaptações no sentido de retardar, assim como atenuar o declínio de diversas funções orgânicas desencadeadas a partir do envelhecimento.
Além disso, a prática do exercício físico é defendida na tentativa de otimizar o desempenho neuromuscular de idosos (Nelson et al., 2004; Ryan et al., 2004). Os exercícios resistidos (ER) vêm sendo apresentados como uma importante estratégia para minimizar os efeitos da sarcopenia, através das adaptações neurais e estruturais decorrentes desta intervenção (Hakkinen, Kraemer, Newton, \& Alen, 2001; Kubo, Kanehisa, Miyatani, Tachi, \& Fukunaga, 2003).

Recentemente, a utilização de estímulos com cargas vibratórias, conhecido como vibração do corpo todo (VCT), tem sido sugerido como uma forma alternativa de intervenção capaz de otimizar a força muscular de idosos (Bogaerts et al., 2007; Machado, Garcia-Lopez, Gonzalez-Gallego, \& Garatachea, 2010; Roelants, Delecluse, \& Verschueren, 2004; Verschueren et al., 2011; Von Stengel, Kemmler, Engelke, \& Kalender, 2012). Diante disso, este estudo teve como objetivo sintetizar, a partir de uma revisão sistemática, as evidências científicas disponíveis sobre a utilização do treino com VCT como uma alternativa para a obtenção de modificações efetivas sobre a força e a potência muscular de idosos.

\section{MÉTODO}

Os estudos selecionados foram recuperados das bases Medline, SciELO, Lilacs, Biblioteca Cochrane, PEDro e Science Citation Index, entre 1982 e dezembro de 2012, a partir da estratégia de busca incluindo a combinação das palavras-chave ("whole body vibration" $O R$ "vibration exercise" OR "vibration training") AND ("muscle strength" OR "strength" OR "force" OR "muscle power" OR "power") AND ("elderly" OR "older"). Os descritores utilizados na base Medline foram: (muscle strength OR strength OR force) AND (elderly OR older) AND (vibration exercise $O R$ vibration training $O R$ whole body vibration) AND (power OR muscle power). Adicionalmente, os seguintes limites foram utilizados para a consulta: acima de 60 anos, humans, english, spanish e portuguese, clinical trial and randomized controlled clinical trial. O período de busca dos 
estudos ocorreu entre 8 de dezembro e 20 de dezembro de 2012.

Dois pesquisadores fizeram a busca de forma independente e cega e posteriormente estes resultados foram confrontados e diante de alguma discordância um terceiro avaliador foi convocado objetivando um consenso. Os critérios de inclusão utilizados para a seleção dos estudos foram: tipo de estudo (ensaio clínico controlado e ensaio clínico controlado e randomizado), artigos disponíveis na íntegra em espanhol, inglês, e português, indivíduos com idade acima de 60 anos e de ambos os sexos e estudos investigando o efeito da intervenção com VCT sobre a força e a potência muscular. Os estudos contendo a comparação dos efeitos da VCT com outros modelos de intervenção também foram incluídos. Foram excluídos os estudos conduzidos em idosos com qualquer diagnóstico de enfermidades neuromusculares e disfunções osteomioarticulares, estudos com proposta de investigar o efeito agudo da VCT, assim como, textos oriundos de artigos de revisão, teses/dissertações, resumos e de congressos.

Seguindo os critérios de elegibilidade, inicialmente foram analisados os títulos e os resumos dos artigos, e aqueles apontados como relevantes foram adquiridos em versão completa para a elaboração de uma análise mais criteriosa. Diante da impossibilidade de obtenção do estudo na íntegra, ainda foi feita uma tentativa de contato com os autores para a sua obtenção, assim como para a possível identificação de estudos adicionais. Referências bibliográficas dos estudos originais recuperados, assim como de revisões da literatura, também foram consultadas objetivando adicionar estudos para a presente revisão.

A análise dos artigos foi conduzida a partir de um roteiro estruturado que considerava os seguintes componentes: característica da amostra, desenho experimental, característica do treinamento vibratório, instrumentos de medida de força e potência muscular e desfechos encontrados. A análise dos dados foi realizada a partir de uma revisão crítica de conteúdo.
A análise da qualidade metodológica dos estudos foi realizada com base na escala PEDro. O instrumento consiste em uma escala com 11 critérios contemplando a análise de validade interna e informações estatísticas para que os resultados dos estudos possam ser interpretados. A pontuação varia de zero a dez, sendo que o critério 1 (validade externa) não é utilizado para calcular a pontuação. Em virtude das características dos ensaios clínicos desta revisão sistemática, ressalta-se a dificuldade de se alcançar certas condições como "cegamento" dos terapeutas ou sujeitos em estudos de intervenção. Optou-se por consultar e manter o escore estabelecido dos estudos selecionados que estavam indexados na base de dados PEDro e que por sua vez já apresentavam a avaliação metodológica. Os escores iguais ou superiores a 5 foram considerados de moderada a alta qualidade (Moseley, Herbert, Sherrington \& Maherz 2002). Para os estudos que não estivessem disponíveis na base de dados PEDro foi realizada uma análise de forma independente por dois investigadores. Em seguida, as diferenças entre os avaliadores foram discutidas e corrigidas.

\section{RESULTADOS}

Dos 91 artigos recuperados, 75 foram excluídos, pois 30 não atenderam aos critérios de inclusão estabelecidos e 45 também foram desconsiderados por serem referências redundantes. Desta forma, foram selecionados 16 estudos para a apreciação crítica quanto aos efeitos do treino de VCT sobre a força e potência muscular de idosos (Figura 1).

Diante da apreciação dos estudos que investigaram o efeito da VCT sobre a força muscular e compararam com a presença de um grupo controle (ausência de intervenção), foram identificados doze estudos (75\%), sendo que $83.3 \%$ destes encontraram que o estímulo vibratório foi superior à condição controle (Tabela 1). Já Vershueren et al. (2011) ao compararem um grupo de VCT associado à suplementação de vitamina $\mathrm{D}$ vs. a condição controle (apenas suplementação de vitamina D) 


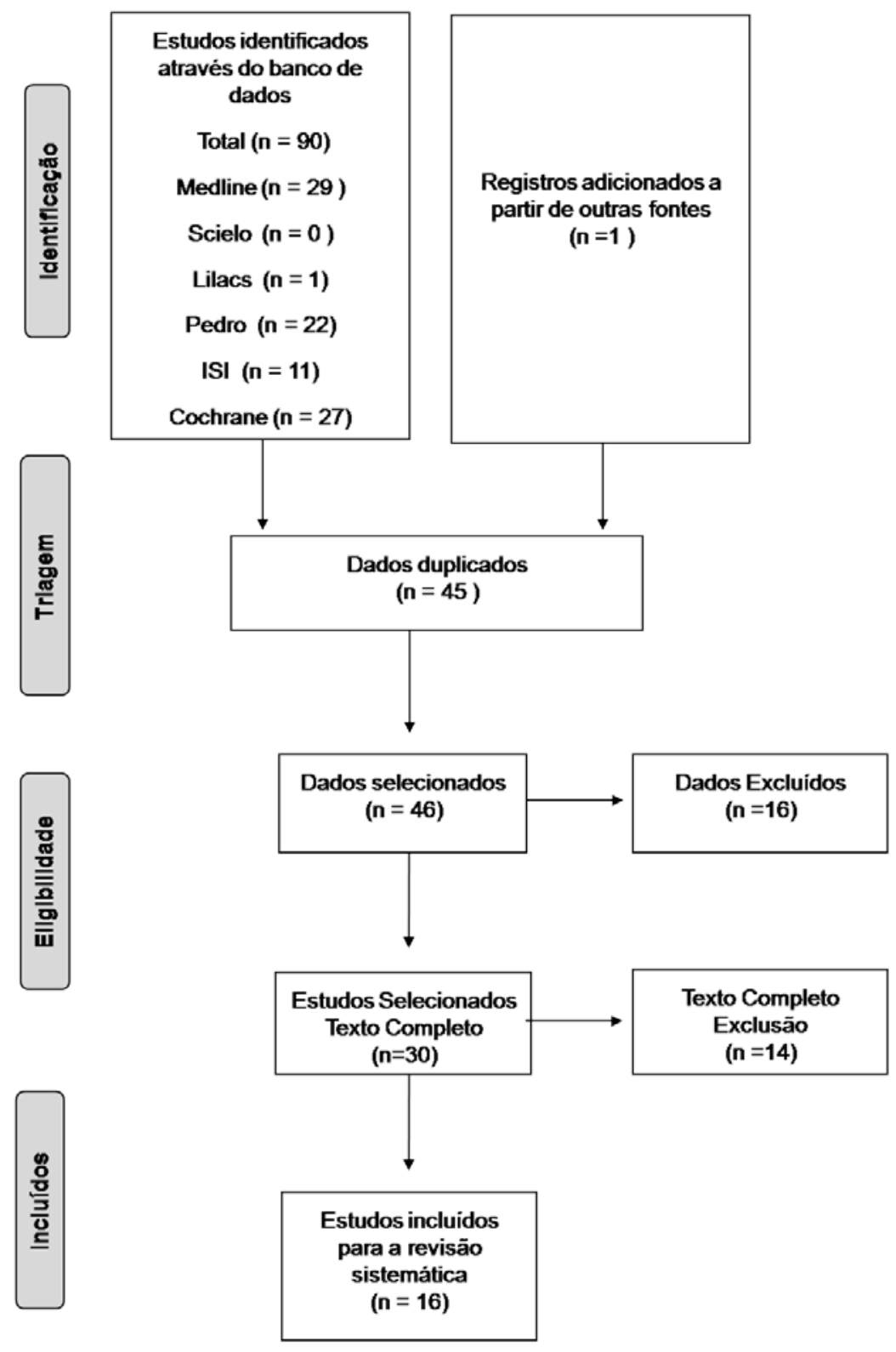

Figura 1. Fluxograma do processo de seleção dos estudos

observaram que ambas as condições promoveram melhora na força muscular dinâmica dos extensores do joelho (6.4 a 7.9\%) após seis meses de intervenção, não indicando efeitos adicionais a partir do estímulo vibratório. De forma similar, Da Silva et al. (2009) não verificaram a presença de efeitos a partir de 13 semanas no grupo de VCT, assim como na condição controle.

Dos 16 artigos elegíveis para compor a presente revisão sistemática, 7 estudos (44\%) compararam a vibração do corpo todo com outra modalidade de exercício físico e os seus respetivos efeitos sobre a força muscular (Tabela 1). Desses estudos, 3 utilizaram os exercícios resistidos (42.8\%), 3 estudos incluíram exercícios de multimodalidades (42.8\%) e apenas um propôs a comparação com exercícios aeróbios (14.4\%) (Tabela 1). Os três estudos que comparam o treino de VCT com as rotinas de exercícios de multimodalidades verificaram que ambas as intervenções promoveram uma melhoria da força muscular isométrica (9.4 a $16.2 \%)$ dos extensores do joelho em 
programas variando de 12 a 18 meses de intervenção, não existindo diferenças entre as abordagens de intervenção. De forma similar, Roelands et al. (2004) e Vershueren et al. (2004) também não observaram diferença nos ganhos de força muscular isométrica e dinâmica dos extensores do joelho, após 24 semanas, entre o grupo de VCT (15 a 16.5\%) quando comparado com o exercício resistido (13.9 a $18.4 \%$ ). Diferentemente, Bemben, Palmer, Bemben e Knehans (2010) verificaram que, após 8 meses, o treino de VCT foi superior na otimização da força muscular dos abdutores (60\%) e adutores (40\%) do quadril quando comparada com a condição de treino de força. Entretanto, não houve nenhuma diferença entre os grupos referente à força muscular dos extensores e flexores do quadril. $\mathrm{O}$ único estudo que propôs um protocolo de exercícios aeróbios versus o treino de VCT não identificou nenhuma modificação na força muscular após 8 meses de intervenção.

Considerando os efeitos sobre a potência muscular, foi identificada a presença de sete estudos, sendo que nenhum efeito foi reportado entre os quatro $(57.1 \%)$ que compararam o treino de VCT com um grupo controle (Tabela 2). Três estudos (42.9\%) compararam as rotinas de VCT com outras modalidades de intervenção, entretanto, apenas Raimundo, Gusi e Tomas-Carus (2009) observaram que o grupo submetido ao treino vibratório obteve um aumento significativo de $7 \%$ na potência muscular, medida pelo salto, quando comparado com o grupo que praticou exercícios aeróbios.

A análise do conteúdo dos estudos selecionados revelou heterogeneidade dos protocolos de intervenção de VCT que identificaram o aumento da força e da potência muscular em idosos. Os efeitos sobre o aumento nas variáveis estudadas foram obtidos em ensaios clínicos envolvendo um período variando de 8 semanas (Rees, Murphy, \& Watsford, 2008) a 18 meses (Von Stengel et al., 2012) de intervenção, com uma frequência entre duas ( $\mathrm{Da}$ Silva et al., 2009; Verschueren et al., 2004; Von Stengel et al., 2012) a cinco sessões
(Machado et al., 2010) semanais. A maioria dos artigos (12 estudos) propôs um programa com uma frequência de três sessões semanais (Bautmans, Van Hees, Lemper, \& Mets, 2005; Bogaerts et al., 2007, 2009; Mikhael, Orr, Amsen, Greene, \& Singh, 2010; Raimundo et al., 2009; Rees et al., 2007, 2008; Roelants et al., 2004; Von Stengel, Kemmler, Bebeneck, Engelke, \& Kalender, 2011), intercaladas por um dia. As intensidades do treino utilizadas variaram de 1 (Mikhael et al., 2010) a $12 \mathrm{~mm}$ (Von Stengel et al., 2011) de amplitude de deslocamento e uma frequência de 12 (Mikhael et al., 2010; Von Stengel et al., 2011) a $40 \mathrm{~Hz}$ (Bautmans et al., 2005; Bogaerts et al., 2007, 2009; Da Silva et al., 2009; Machado et al., 2010; Roelants et al., 2004; Verschueren et al., 2004). Grande parte dos estudos (43.7\%) adotou como procedimento a realização de 30 a 40 $\mathrm{Hz}$ de frequência. $\mathrm{O}$ volume típico encontrado, considerando a duração do estímulo de VCT, incluiu protocolos de 30 (Bautmans et al., 2005; Bogaerts et al., 2007, 2009; Da Silva et al., 2009; Machado et al., 2010) a 90 segundos (Von Stengel et al., 2011).

O tamanho da amostra e a idade média dos participantes variaram de 19 (Mikhael et al., 2010) a 214 idosos (Bogaerts et al., 2009) e de 62.3 (Mikhael et al., 2010) a 79.3 anos (Machado et al., 2010), respetivamente.

Dentre as formas de medida de força muscular estão: o teste de uma repetição máxima (1RM) (Bemben et al., 2010; Mikhael et al., 2010), dinamómetro isocinético (Bautmans et al., 2005; Bogaerts et al., 2007, 2009; Da Silva et al., 2009; Raimundo et al., 2009; Rees et al., 2007, 2008; Roelants et al., 2004; Verschueren et al., 2004) e plataforma de força (Machado et al., 2010; Von Stengel et al., 2011, 2012). A força muscular dinâmica foi avaliada em 10 estudos (Bautmans et al., 2005; Bemben et al., 2010; Da Silva et al., 2009; Mikhael et al., 2010; Raimundo et al., 2009; Rees et al., 2007, 2008; Roelants et al., 2004; Verschueren et al., 2004, 2011) e a isométrica em 9 estudos (Bogaerts et al., 2007, 2009, 2011; Machado et al., 2010; Roelants et al., 2004; Verschueren et 
Tabela 1

Efeitos do treino com VCT sobre a força muscular de idosos saudáveis

\begin{tabular}{|c|c|c|c|c|c|c|c|c|c|c|}
\hline \multirow{2}{*}{$\begin{array}{l}\text { Autor } \\
\text { (data) }\end{array}$} & \multirow{2}{*}{$\begin{array}{l}\text { Sujeitos } \\
\text { (Idade) }\end{array}$} & \multirow[b]{2}{*}{ Grupos } & \multicolumn{3}{|c|}{ Característica da Vibração } & \multirow[b]{2}{*}{ Exercícios } & \multirow{2}{*}{$\begin{array}{l}\text { Duração/ } \\
\text { Frequência } \\
\text { semanal }\end{array}$} & \multicolumn{2}{|c|}{ Desempenho Muscular } & \multirow{2}{*}{$\begin{array}{l}\text { Escala } \\
\text { PEDro }\end{array}$} \\
\hline & & & $\begin{array}{l}\text { Amp } \\
(\mathrm{mm})\end{array}$ & $\begin{array}{l}\text { Freq } \\
(\mathrm{Hz})\end{array}$ & $\begin{array}{l}\text { Tempo de } \\
\text { Exposição } \\
\text { VIB }\end{array}$ & & & Avaliação & Resultados & \\
\hline \multirow{3}{*}{$\begin{array}{l}\text { Von Sten- } \\
\text { gel et al. } \\
(2012)\end{array}$} & $\begin{array}{c}50 \mathrm{M} \\
(68.8 \pm 3.6)\end{array}$ & $\mathrm{EX}+\mathrm{VCT}$ & \multirow{3}{*}{$1.7-2.0$} & \multirow{3}{*}{$25-35$} & \multirow{3}{*}{$\begin{array}{c}\text { 60s } \\
\text { DT: } 6 \mathrm{~min}\end{array}$} & $\begin{array}{c}\mathrm{EM}+\mathrm{ER} \\
\mathrm{c} / \mathrm{VCT}\end{array}$ & \multirow{3}{*}{$\begin{array}{l}18 \text { meses } \\
2 \mathrm{x} \text { sem }\end{array}$} & \multirow{3}{*}{$\begin{array}{l}\text { Leg Press } \\
\text { (PF) } \\
\text { FM Isom } \\
\text { Pré /Pós }\end{array}$} & \multirow{3}{*}{$\begin{array}{c}\text { EX+VCT: FM Isom } * \mathrm{~F} \uparrow 16.2 \% \\
\text { EX: FM Isom } * \uparrow 11.8 \% \\
\text { CON: NS }\end{array}$} & \multirow{3}{*}{$7 / 10$} \\
\hline & $\begin{array}{c}50 \mathrm{M} \\
(68.6 \pm 3.0)\end{array}$ & EX & & & & $\begin{array}{l}\mathrm{EM}+\mathrm{ER} \\
\mathrm{s} / \mathrm{VCT}\end{array}$ & & & & \\
\hline & $\begin{array}{c}51 \mathrm{M} \\
(68.1 \pm 2.7)\end{array}$ & $\mathrm{CON}$ & & & & Relaxamento & & & & \\
\hline \multirow{2}{*}{$\begin{array}{l}\text { Bogaerts et } \\
\text { al. (2011) }\end{array}$} & $\begin{array}{l}54 \mathrm{MI} \\
(80.0)\end{array}$ & & \multirow{2}{*}{$\begin{array}{l}1.6 \mathrm{a} \\
2.2 \mathrm{~g}\end{array}$} & \multirow{2}{*}{$30-40$} & \multirow{2}{*}{$\begin{array}{l}15-60 s \\
\text { NR n } \\
\text { series }\end{array}$} & $5 \mathrm{ED}$ & \multirow{2}{*}{$\begin{array}{l}6 \text { meses } \\
3 x \text { sem }\end{array}$} & \multirow{2}{*}{$\begin{array}{l}\text { FM Isom } \\
\text { Máx } \\
\text { EJ } \\
\text { (NR) }\end{array}$} & \multirow{2}{*}{$\begin{array}{c}\text { VCT: }{ }^{*}{ }^{\mp} \uparrow 14.9 \% \\
\text { CON: ND }\end{array}$} & \multirow{2}{*}{$7 / 10$} \\
\hline & $\begin{array}{l}57 \mathrm{MI} \\
(79.1) \\
\end{array}$ & $\mathrm{CON}$ & & & & & & & & \\
\hline \multirow{2}{*}{$\begin{array}{l}\text { Verschue- } \\
\text { ren et al. } \\
(2011) \text {. }\end{array}$} & $\begin{array}{l}54 \mathrm{M} \mathrm{I} \\
(80.1)\end{array}$ & $\begin{array}{c}\mathrm{VCT}+\mathrm{VIT} \\
\mathrm{D}\end{array}$ & \multirow{2}{*}{$\begin{array}{l}1.6 \mathrm{a} \\
2.2 \mathrm{~g}\end{array}$} & \multirow{2}{*}{$30-40$} & \multirow{2}{*}{$\begin{array}{c}\text { 15-60s } \\
\text { 3-5 séries }\end{array}$} & $\begin{array}{l}\text { EE/ED + } \\
\text { VITD }\end{array}$ & \multirow{2}{*}{$\begin{array}{l}6 \text { meses } \\
3 x \text { sem }\end{array}$} & \multirow{2}{*}{$\begin{array}{c}\text { FM Isom } \\
\text { FM Din } \\
\text { (Dinamô- } \\
\text { metro) } \\
\text { EJ } \\
\text { Pré /Pós }\end{array}$} & $\begin{array}{l}\text { VCT: FM Isom: NS } \\
\text { CON: FM Isom: NS }\end{array}$ & \multirow{2}{*}{$7 / 10$} \\
\hline & $\begin{array}{l}57 \mathrm{M} \mathrm{I} \\
(79.2)\end{array}$ & $\begin{array}{c}\mathrm{CON}+\mathrm{VI} \\
\mathrm{TD}\end{array}$ & & & & VITD & & & $\begin{array}{l}\text { VCT: FM Din: } \uparrow^{*} 7.9 \% \\
\text { CON:FM Din: } \uparrow^{*} 6.4 \%\end{array}$ & \\
\hline \multirow{4}{*}{$\begin{array}{l}\text { Von Sten- } \\
\text { gel et al. } \\
(2011)\end{array}$} & $68.1 \pm 4.0 \mathrm{M}$ & VCT & \multirow{4}{*}{$\begin{array}{l}1.7 \\
12\end{array}$} & \multirow{4}{*}{$\begin{array}{l}35 \\
12\end{array}$} & & ED & \multirow{4}{*}{$\begin{array}{l}12 \text { meses } \\
3 \mathrm{x} \text { sem }\end{array}$} & \multirow{4}{*}{$\begin{array}{l}\text { LegPress } \\
\text { FM Isom } \\
\quad(\mathrm{PF})\end{array}$} & \multirow{4}{*}{$\begin{array}{c}\mathrm{V}-{ }^{* \mathrm{~F}} \uparrow 24.4 \% \\
\mathrm{R}-{ }^{* \mathrm{~F}} \uparrow 26.6 \% \\
\mathrm{CON}-\mathrm{NS}\end{array}$} & \\
\hline & $679+38 \mathrm{M}$ & VCT & & & $90 s$ & ED & & & & $8 / 10^{\mathbb{s}}$ \\
\hline & & & & & & & & & & \\
\hline & $67.6 \pm 4.1 \mathrm{M}$ & $\mathrm{CON}$ & & & & Relaxamento & & & & \\
\hline
\end{tabular}

Nota: A- ativos; CON- controle; DT - duração total; ED- Exercício dinâmico; EE - exercício estático; EJ- extensão do joelho; EM - exercícios multimodalidades (Aeróbio + Equilíbrio +

Treino Resistido (Membros superiores e Tronco); ER- exercício resistido; EX - exercício; EX + VCT - exercício + VCT; FJ - flexão do joelho; FM Din - força muscular dinâmica; FM Isom força muscular isométrica; H- Homem; I - Institucionalizados; M - mulher; MMSS - membro superior; MMII - membro inferior; NR- não reportado; NS- não significativo; PF- Plataforma de força; R- Rotacional; RM -repetição máxima; S- segundos; Sem - semanas; V - vertical; VIT D- vitamina D; *diferença significativa quando comparada com os valores pré-intervenção;

${ }^{\mp}$ diferença significativa quando comparada com os valores do grupo controle, ${ }^{++}$diferença significativa quando comparada com os valores do grupo de ER/EM; ${ }^{\S}$ estudos que não estavam indexados na base PEDro para a análise metodológica. 
Tabela 1 (continuação)

Efeitos do treino com VCT sobre a força muscular de idosos saudáveis

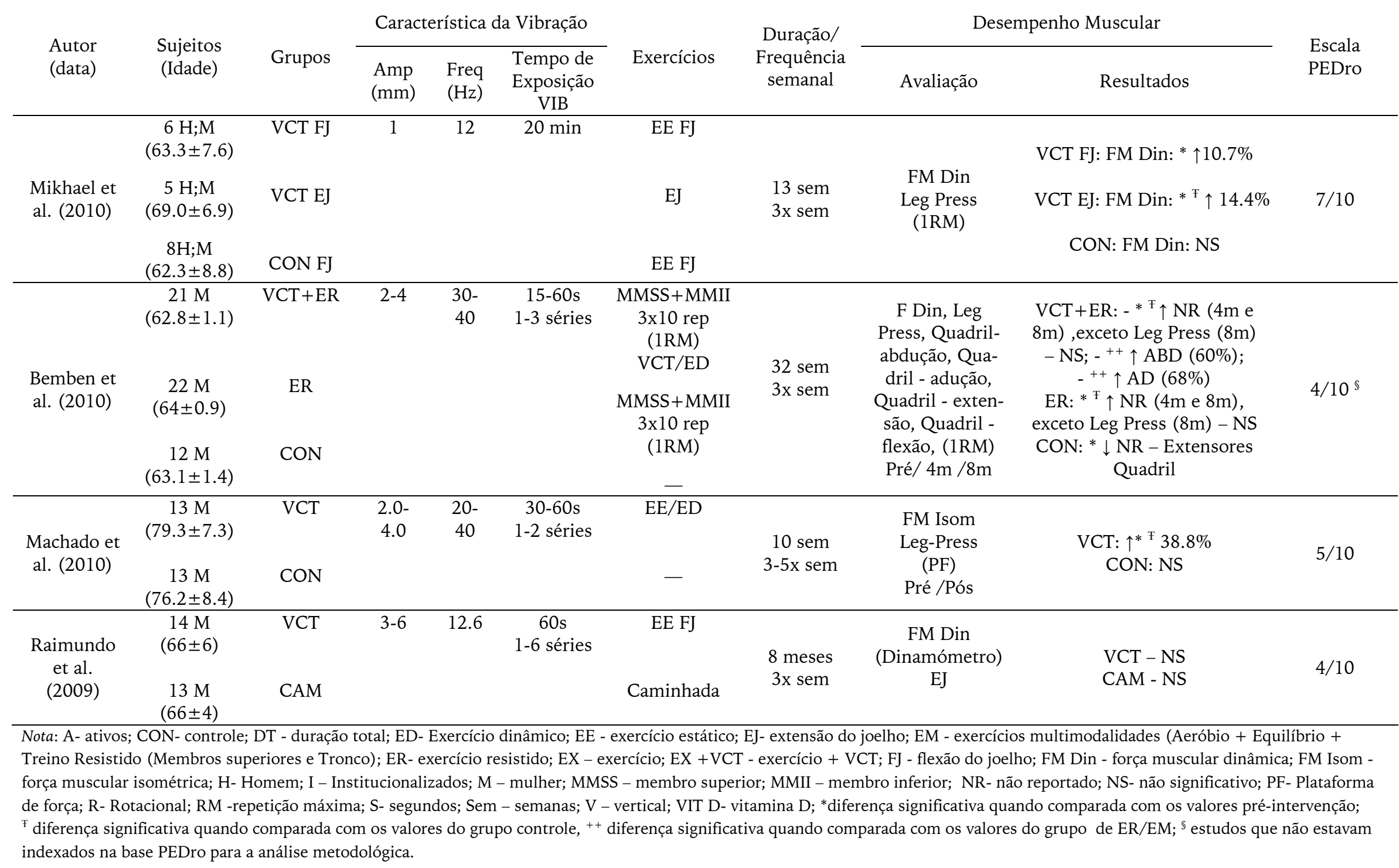


Tabela 1 (continuação)

Efeitos do treino com VCT sobre a força muscular de idosos saudáveis

\begin{tabular}{|c|c|c|c|c|c|c|c|c|c|c|}
\hline \multirow[b]{2}{*}{$\begin{array}{l}\text { Autor } \\
\text { (data) }\end{array}$} & \multirow[b]{2}{*}{$\begin{array}{l}\text { Sujeitos } \\
\text { (Idade) }\end{array}$} & \multirow[b]{2}{*}{ Grupos } & \multicolumn{3}{|c|}{ Característica da Vibração } & \multirow[b]{2}{*}{ Exercícios } & \multirow{2}{*}{$\begin{array}{l}\text { Duração/ } \\
\text { Frequência } \\
\text { semanal }\end{array}$} & \multicolumn{2}{|r|}{ Desempenho Muscular } & \multirow[b]{2}{*}{$\begin{array}{l}\text { Escala } \\
\text { PEDro }\end{array}$} \\
\hline & & & $\begin{array}{l}\text { Amp } \\
(\mathrm{mm})\end{array}$ & $\begin{array}{l}\text { Freq } \\
(\mathrm{Hz})\end{array}$ & $\begin{array}{l}\text { Tempo de } \\
\text { Exposição } \\
\text { VIB }\end{array}$ & & & Avaliação & Resultados & \\
\hline \multirow{3}{*}{$\begin{array}{l}\text { Bogaerts et } \\
\text { al. }(2009)\end{array}$} & $\begin{array}{c}92 \mathrm{M} ; \mathrm{H} \\
(66.8 \pm 0.5)\end{array}$ & VCT & $2.5-5.0$ & $30-40$ & $\begin{array}{l}\text { 30-60s } \\
1-3 \text { séries }\end{array}$ & $\mathrm{EE} / \mathrm{ED}$ & \multirow{3}{*}{$\begin{array}{l}12 \text { meses } \\
3 \mathrm{x} \text { sem }\end{array}$} & $\begin{array}{l}\text { FM Isom } \\
\text { (Dinamó- }\end{array}$ & VCT: FM Isom: ${ }^{* \mp} \uparrow 9.4 \%$ & \multirow{3}{*}{$6 / 10$} \\
\hline & $\begin{array}{c}57 \mathrm{M} ; \mathrm{H} \\
(66.5 \pm 0.6)\end{array}$ & EM & & & & EM & & $\begin{array}{l}\text { metro) } \\
\text { EJ }\end{array}$ & FIT: FM Isom: ${ }^{* \mp} \uparrow 12.5 \%$ & \\
\hline & $\begin{array}{c}65 \mathrm{M} ; \mathrm{H} \\
(67.6 \pm 0.6)\end{array}$ & $\mathrm{CON}$ & & & & - & & Pré / Pós & CON: FM Isom: NS & \\
\hline \multirow{3}{*}{$\begin{array}{l}\text { Da Silva et } \\
\text { al. (2009) }\end{array}$} & $\begin{array}{c}24 \mathrm{M} ; \mathrm{H} \mathrm{A} \\
(70.7 \pm 5.8)\end{array}$ & VCT & $2.0-6.0$ & $30-40$ & $\begin{array}{c}\text { 30-60s } \\
1-3 \text { séries }\end{array}$ & EE & $13 \mathrm{sem}$ & $\begin{array}{l}\text { FM Din } \\
\text { (Dinamó- } \\
\text { metro) }\end{array}$ & \multirow{3}{*}{ NS } & \multirow{3}{*}{$4 / 10 \$$} \\
\hline & $23 \mathrm{M} ; \mathrm{H} \mathrm{A}$ & $\mathrm{CON}$ & & & & & $2 \mathrm{x} \mathrm{sem}$ & EJ & & \\
\hline & $(70.0 \pm 5.7)$ & & & & & - & & Pré /Pós & & \\
\hline \multirow[b]{2}{*}{$\begin{array}{l}\text { Rees et al. } \\
\quad(2008)\end{array}$} & $\begin{array}{c}15 \mathrm{H} ; \mathrm{M} \\
(74,3 \pm 5,0)\end{array}$ & VCT & 8 & 26 & $\begin{array}{l}45-80 \mathrm{~s} \\
6 \text { séries }\end{array}$ & EE/ED & \multirow[b]{2}{*}{$\begin{array}{c}8 \text { sem } \\
3 x \text { sem }\end{array}$} & $\begin{array}{l}\text { FM Din } \\
\text { F/E Qua- } \\
\text { dril, Joelho }\end{array}$ & $\begin{array}{c}\text { Quadril: VCT e CON- NS } \\
\text { Joelho: VCT- (F e E) - * } \uparrow 8.0- \\
9.6 \%\end{array}$ & \\
\hline & $\begin{array}{c}15 \mathrm{H} ; \mathrm{M} \\
(73.1 \pm 4.1)\end{array}$ & $\mathrm{CON}$ & & & & $\mathrm{EE} / \mathrm{ED}$ & & $\begin{array}{l}\text { e Tornozelo } \\
\text { (Dinamó- } \\
\text { metro) } \\
\text { Pré / Pós }\end{array}$ & $\begin{array}{c}\text { CON - }(\mathrm{F} \mathrm{e} \mathrm{E})-{ }^{*} \uparrow 6.1-6.7 \% \\
\text { Tornozelo: VCT }(\mathrm{FP}):{ }^{* \mathrm{~F}} \uparrow 18.2 \text {; } \\
\text { (DF): NS } \\
\text { CON - (FP) - NS; (DF): NS }\end{array}$ & $5 / 10$ \\
\hline \multirow{3}{*}{$\begin{array}{l}\text { Bogaerts et } \\
\text { al. }(2007)\end{array}$} & $\begin{array}{c}31 \mathrm{M} ; \mathrm{H} \\
(67.3 \pm 0.7)\end{array}$ & VCT & $2.5-5.0$ & $30-40$ & $\begin{array}{c}\text { 30-60s } \\
1-3 \text { séries }\end{array}$ & EE/ED & \multirow{3}{*}{$\begin{array}{l}12 \text { meses } \\
3 \mathrm{x} \text { sem }\end{array}$} & $\begin{array}{l}\text { FM Isom } \\
\text { (Dinamó- }\end{array}$ & VCT: FM Isom: ${ }^{* \mp} \uparrow 9.8 \%$ & \multirow{3}{*}{$4 / 10$} \\
\hline & $\begin{array}{c}30 \mathrm{M} ; \mathrm{H} \\
(67.4 \pm 0.8)\end{array}$ & EM & & & & EM & & $\begin{array}{l}\text { metro) } \\
\text { EJ }\end{array}$ & RES: FM Isom: ${ }^{* \mp} \uparrow 13.1 \%$ & \\
\hline & $\begin{array}{c}36 \mathrm{M} ; \mathrm{H} \\
(68.6 \pm 0.9)\end{array}$ & $\mathrm{CON}$ & & & & - & & Pré / Pós & CON: FM Isom: NS & \\
\hline
\end{tabular}

Nota: A- ativos; CON- controle; DT - duração total; ED- Exercício dinâmico; EE - exercício estático; EJ- extensão do joelho; EM - exercícios multimodalidades (Aeróbio + Equilíbrio +

Treino Resistido (Membros superiores e Tronco); ER- exercício resistido; EX - exercício; EX +VCT - exercício + VCT; FJ - flexão do joelho; FM Din - força muscular dinâmica; FM Isom força muscular isométrica; H- Homem; I - Institucionalizados; M - mulher; MMSS - membro superior; MMII - membro inferior; NR- não reportado; NS- não significativo; PF- Plataforma de força; R- Rotacional; RM -repetição máxima; S- segundos; Sem - semanas; V - vertical; VIT D- vitamina D; *diferença significativa quando comparada com os valores pré-intervenção;

${ }^{\mathrm{F}}$ diferença significativa quando comparada com os valores do grupo controle, ${ }^{++}$diferença significativa quando comparada com os valores do grupo de ER/EM; ${ }^{\circledR}$ estudos que não estavam indexados na base PEDro para a análise metodológica. 
Tabela 1 (continuação)

Efeitos do treino com VCT sobre a força muscular de idosos saudáveis

\begin{tabular}{|c|c|c|c|c|c|c|c|c|c|c|}
\hline \multirow{2}{*}{$\begin{array}{l}\text { Autor } \\
\text { (data) }\end{array}$} & \multirow{2}{*}{$\begin{array}{l}\text { Sujeitos } \\
\text { (Idade) }\end{array}$} & \multirow[b]{2}{*}{ Grupos } & \multicolumn{3}{|c|}{ Característica da Vibração } & \multirow[b]{2}{*}{ Exercícios } & \multirow{2}{*}{$\begin{array}{l}\text { Duração/ } \\
\text { Frequência } \\
\text { semanal }\end{array}$} & \multicolumn{2}{|r|}{ Desempenho Muscular } & \multirow{2}{*}{$\begin{array}{l}\text { Escala } \\
\text { PEDro }\end{array}$} \\
\hline & & & $\begin{array}{l}\text { Amp } \\
(\mathrm{mm})\end{array}$ & $\begin{array}{l}\text { Freq } \\
(\mathrm{Hz})\end{array}$ & $\begin{array}{c}\text { Tempo de } \\
\text { Exposição } \\
\text { VIB }\end{array}$ & & & Avaliação & Resultados & \\
\hline $\begin{array}{l}\text { Rees et al. } \\
\text { (2007) }\end{array}$ & $\begin{array}{c}15 \mathrm{H} ; \mathrm{M} \\
74.3 \pm 5.0 \\
13 \mathrm{H} ; \mathrm{M} \\
73.1 \pm 4.1 \\
15 \mathrm{H} ; \mathrm{M} \\
73.1 \pm 4.6 \\
\end{array}$ & $\mathrm{EX}+\mathrm{VCT}$ & $5-8$ & 26 & $\begin{array}{l}45-80 s \\
6 \text { séries }\end{array}$ & EE/ED & $\begin{array}{l}2 \text { meses } \\
3 \mathrm{x} \text { sem }\end{array}$ & $\begin{array}{c}\text { FM Din } \\
\text { Quadril - } \\
\text { F/E } \\
\text { Joelho - F/E } \\
\text { Tornozelo - } \\
\text { F/E } \\
\text { (Dinamóme- } \\
\text { tro) } \\
\end{array}$ & 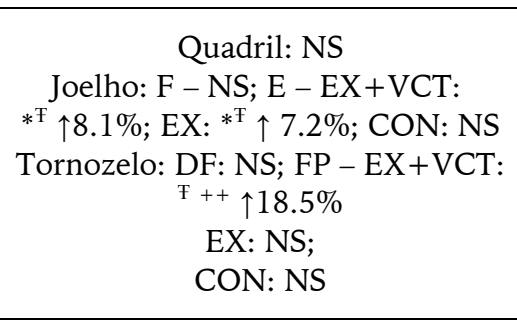 & $5 / 10$ \\
\hline $\begin{array}{l}\text { Bautmans et } \\
\text { al. (2005) }\end{array}$ & $\begin{array}{c}10 \mathrm{H} ; \mathrm{M} \mathrm{I} \\
(76.3 \pm 9.7) \\
11 \mathrm{H} ; \mathrm{M} \mathrm{I} \\
(78.6 \pm 10.4)\end{array}$ & $\mathrm{CON}$ & $2-5$ & $35-40$ & $\begin{array}{c}\text { 30-60s } \\
1-3 \text { séries }\end{array}$ & $\mathrm{EE}$ & $\begin{array}{c}6 \mathrm{sem} \\
3 \mathrm{x} \mathrm{sem}\end{array}$ & $\begin{array}{c}\text { FM Din } \\
\text { (Dinamó- } \\
\text { metro) } \\
\text { EJ } \\
\text { Pré / Pós } \\
\end{array}$ & $\begin{array}{l}\text { VCT: NS } \\
\text { CON: NS }\end{array}$ & $7 / 10$ \\
\hline $\begin{array}{l}\text { Roelands et } \\
\text { al. (2004). }\end{array}$ & $\begin{array}{c}24 \mathrm{M} \\
(64.6 \pm 0.7) \\
\\
20 \mathrm{M} \\
(63.9 \pm 0.8) \\
25 \mathrm{M} \\
(64.2 \pm 0.6) \\
\end{array}$ & $\mathrm{CON}$ & $2.5-5.5$ & $35-40$ & $\begin{array}{c}\text { 30-60s } \\
1-3 \text { series }\end{array}$ & Aeróbio+ ER & $\begin{array}{l}24 \text { sem } \\
3 x \text { sem }\end{array}$ & $\begin{array}{l}\text { FM Isom } \\
\text { FM Din } \\
\text { (Dinamó- } \\
\text { metro) } \\
\text { EJ } \\
\text { Pré } / 12 \\
\text { sem } / 24 \text { sem }\end{array}$ & $\begin{array}{c}\text { VCT: FM Isom: }{ }^{* \mp} \uparrow 15.0 \% \\
\text { ER: FM Isom: }{ }^{\mp} \uparrow 18.4 \% \\
\text { CON: FM Isom: NS } \\
\text { VCT: FM Din: }{ }^{* \mp} \uparrow 16.1 \% \\
\text { ER: FM Din: }{ }^{* \mathrm{~F}} \uparrow 13.9 \% \\
\text { CON: FM Din: NS }\end{array}$ & $4 / 10$ \\
\hline $\begin{array}{l}\text { Verschueren } \\
\text { et al. (2004) }\end{array}$ & $\begin{array}{c}25 \mathrm{M} \\
(64.6 \pm 3.3) \\
22 \mathrm{M} \\
(63.9 \pm 3.8) \\
24 \mathrm{M} \\
(64.2 \pm 3.1)\end{array}$ & $\mathrm{CON}$ & $1.7-2.5$ & $35-40$ & NR & $\begin{array}{c}\text { EE/ED } \\
\text { Exer. aeróbio } \\
\text { Leg-Press } \\
\text { Cad Extensora }\end{array}$ & $\begin{array}{l}24 \text { sem } \\
2 x \text { sem }\end{array}$ & $\begin{array}{l}\text { FM Isom } \\
\text { FM Din } \\
\text { (Dinamó- } \\
\text { metro) } \\
\text { EJ }\end{array}$ & $\begin{array}{c}\text { VCT: FM Isom: }{ }^{* \mathrm{~F}} \uparrow 15 \% \\
\text { ER: FM Isom: }{ }^{*} \uparrow 16 \% \\
\text { CON: FM Isom: NS } \\
\text { VCT: FM Din: }{ }^{* \mathrm{~F}} \uparrow 16.5 \% \\
\text { ER: FM Din: }{ }^{* \mathrm{~F}} \uparrow 10.6 \% \\
\text { CON: FM Din: NS }\end{array}$ & $5 / 10$ \\
\hline
\end{tabular}

Nota: A- ativos; CON- controle; DT - duração total; ED- Exercício dinâmico; EE - exercício estático; EJ- extensão do joelho; EM - exercícios multimodalidades (Aeróbio + Equilíbrio + Treino Resistido (Membros superiores e Tronco); ER- exercício resistido; EX - exercício; EX +VCT - exercício + VCT; FJ - flexão do joelho; FM Din - força muscular dinâmica; FM Isom força muscular isométrica; H- Homem; I - Institucionalizados; M - mulher; MMSS - membro superior; MMII - membro inferior; NR- não reportado; NS- não significativo; PF- Plataforma de força; R- Rotacional; RM -repetição máxima; S- segundos; Sem - semanas; V - vertical; VIT D- vitamina D; *diferença significativa quando comparada com os valores pré-intervenção; ${ }^{\mathrm{F}}$ diferença significativa quando comparada com os valores do grupo controle, ${ }^{++}$diferença significativa quando comparada com os valores do grupo de ER/EM; ${ }^{\S}$ estudos que não estavam indexados na base PEDro para a análise metodológica. 
Tabela 2

Efeitos do treino com VCT sobre a potência muscular de idosos saudáveis

\begin{tabular}{|c|c|c|c|c|c|c|c|c|c|c|}
\hline \multirow[b]{2}{*}{$\begin{array}{l}\text { Autor } \\
\text { (data) }\end{array}$} & \multirow[b]{2}{*}{$\begin{array}{l}\text { Sujeitos } \\
\text { (Idade) }\end{array}$} & \multirow[b]{2}{*}{ Grupos } & \multicolumn{3}{|c|}{ Característica da Vibração } & \multirow[b]{2}{*}{ Exercícios } & \multirow[b]{2}{*}{$\begin{array}{l}\text { Duração/ } \\
\text { Frequência } \\
\text { semanal }\end{array}$} & \multicolumn{2}{|c|}{ Desempenho Muscular } & \multirow[b]{2}{*}{$\begin{array}{l}\text { Escala } \\
\text { PEDro }\end{array}$} \\
\hline & & & $\begin{array}{l}\text { Amp } \\
(\mathrm{mm})\end{array}$ & $\begin{array}{l}\text { Freq } \\
(\mathrm{Hz})\end{array}$ & $\begin{array}{c}\text { Tempo de } \\
\text { Exposição } \\
\text { VIB } \\
\end{array}$ & & & Avaliação & Resultados & \\
\hline \multirow{3}{*}{$\begin{array}{l}\text { Von Sten- } \\
\text { gel et al. } \\
(2012)\end{array}$} & $\begin{array}{c}50 \mathrm{M} \\
(68.8 \pm 3.6)\end{array}$ & $\mathrm{EX}+\mathrm{VCT}$ & $1.7-2.0$ & $25-35$ & $60 s$ & $\mathrm{EM}+\mathrm{ER} \mathrm{c} / \mathrm{VCT}$ & & & & \\
\hline & $\begin{array}{c}50 \mathrm{M} \\
(68.6 \pm 3.0)\end{array}$ & EX & & & & $\mathrm{EM}+\mathrm{ER} \mathrm{s} / \mathrm{VCT}$ & $\begin{array}{l}18 \text { meses } \\
2 x \text { sem }\end{array}$ & Salto & NS & $7 / 10$ \\
\hline & $\begin{array}{c}51 \mathrm{M} \\
(68.1 \pm 2.7)\end{array}$ & $\mathrm{CON}$ & & & & Relaxamento & & & & \\
\hline \multirow{3}{*}{$\begin{array}{l}\text { Von Sten- } \\
\text { gel et al. } \\
(2011)\end{array}$} & $68.1 \pm 4.0 \mathrm{M}$ & VCT & 1.7 & 35 & $90 \mathrm{~s}$ & ED & 12 meses & & & \\
\hline & $67.9 \pm 3.8 \mathrm{M}$ & VCT & 12 & 12 & & ED & $3 x$ sem & Salto & NS & $7 / 10$ \\
\hline & $67.6 \pm 4.1 \mathrm{M}$ & $\mathrm{CON}$ & & & & Relaxamento & & & & \\
\hline \multirow[t]{3}{*}{$\begin{array}{l}\text { Mikhael et } \\
\text { al. (2010) }\end{array}$} & $\begin{array}{c}6 \mathrm{H} ; \mathrm{M} \\
(63.3 \pm 7.6)\end{array}$ & VCT FJ & 1 & 12 & $20 \mathrm{~min}$ & EE FJ & & & & \\
\hline & $\begin{array}{c}5 \mathrm{H} ; \mathrm{M} \\
(69.0 \pm 6.9)\end{array}$ & VCT EJ & & & $\begin{array}{c}\mathrm{VCT}+1 \\
\text { min } \\
\text { intervalo) }\end{array}$ & EJ & $\begin{array}{l}13 \text { sem } \\
3 x \text { sem }\end{array}$ & NR & NS & $7 / 10$ \\
\hline & $\begin{array}{c}8 \mathrm{H} ; \mathrm{M} \\
(62.3 \pm 8.8)\end{array}$ & $\mathrm{CON} \mathrm{FJ}$ & & & & EE FJ & & & & \\
\hline \multirow[t]{2}{*}{$\begin{array}{l}\text { Machado et } \\
\text { al. (2010) }\end{array}$} & $\begin{array}{c}13 \mathrm{M} \\
(79.3 \pm 7.3)\end{array}$ & VCT & $2.0-4.0$ & $20-40$ & $\begin{array}{c}30-60 \mathrm{~s} \\
1-2 \text { séries }\end{array}$ & $\begin{array}{c}\text { EE/ED } \\
\text { Aquecimento e } \\
\text { Relaxamento }\end{array}$ & $\begin{array}{c}10 \text { sem } \\
3-5 x \text { sem }\end{array}$ & $\begin{array}{c}\text { Dinamómetro } \\
\text { EJ }\end{array}$ & NS & $5 / 10$ \\
\hline & $\begin{array}{c}13 \mathrm{M} \\
(76.2 \pm 8.4)\end{array}$ & $\mathrm{CON}$ & & & & - & & & & \\
\hline \multirow{2}{*}{$\begin{array}{l}\text { Raimundo } \\
\text { et al. } \\
(2009)\end{array}$} & $\begin{array}{c}14 \mathrm{M} \\
(66 \pm 6)\end{array}$ & VCT & $3-6$ & 12.6 & $\begin{array}{c}60 \mathrm{~s} \\
1-6 \text { séries }\end{array}$ & EE FJ & $\begin{array}{l}32 \text { sem } \\
3 x \text { sem }\end{array}$ & Salto & $\begin{array}{c}\text { VCT: }{ }^{++} \uparrow 7 \% \\
\text { CAM: NS }\end{array}$ & \\
\hline & $\begin{array}{c}13 \mathrm{M} \\
(66 \pm 4)\end{array}$ & CAM & & & & Caminhada & & & & $4 / 10$ \\
\hline
\end{tabular}


Tabela 2 (continuação)

Efeitos do treino com VCT sobre a potência muscular de idosos saudáveis

\begin{tabular}{|c|c|c|c|c|c|c|c|c|c|c|}
\hline \multirow[b]{2}{*}{$\begin{array}{l}\text { Autor } \\
\text { (data) }\end{array}$} & \multirow[b]{2}{*}{$\begin{array}{l}\text { Sujeitos } \\
\text { (Idade) }\end{array}$} & \multirow[b]{2}{*}{ Grupos } & \multicolumn{3}{|c|}{ Característica da Vibração } & \multirow[b]{2}{*}{ Exercícios } & \multirow[b]{2}{*}{$\begin{array}{l}\text { Duração/ } \\
\text { Frequência } \\
\text { semanal }\end{array}$} & \multicolumn{2}{|c|}{ Desempenho Muscular } & \multirow[b]{2}{*}{$\begin{array}{l}\text { Escala } \\
\text { PEDro }\end{array}$} \\
\hline & & & $\begin{array}{l}\text { Amp } \\
(\mathrm{mm})\end{array}$ & $\begin{array}{l}\text { Freq } \\
(\mathrm{Hz})\end{array}$ & $\begin{array}{c}\text { Tempo de } \\
\text { Exposição } \\
\text { VIB }\end{array}$ & & & Avaliação & Resultados & \\
\hline \multirow[b]{2}{*}{$\begin{array}{l}\text { Da Silva et } \\
\text { al. (2009) }\end{array}$} & $\begin{array}{c}24 \mathrm{M} ; \mathrm{H} \mathrm{A} \\
(70.7 \pm 5.8)\end{array}$ & VCT & $2.0-6.0$ & $30-40$ & $\begin{array}{c}\text { 30-60s } \\
1-3 \text { séries }\end{array}$ & $\mathrm{EE}$ & & \multirow[b]{2}{*}{$\begin{array}{c}\text { Dinamómetro } \\
\text { EJ }\end{array}$} & \multirow[b]{2}{*}{ NS } & \multirow[b]{2}{*}{$4 / 10$} \\
\hline & $\begin{array}{c}23 \mathrm{M} ; \mathrm{H} \mathrm{A} \\
(70.0 \pm 5.7)\end{array}$ & $\mathrm{CON}$ & & & & - & $\begin{array}{l}13 \mathrm{sem} \\
2 \mathrm{x} \text { sem }\end{array}$ & & & \\
\hline $\begin{array}{l}\text { Rees et al. } \\
\quad(2008)\end{array}$ & $\begin{array}{c}15 \mathrm{H} ; \mathrm{M} \\
(74.3 \pm 5.0)\end{array}$ & $\mathrm{CON}$ & $5-8$ & 26 & $\begin{array}{l}45-80 \text { s } \\
6 \text { séries }\end{array}$ & $\mathrm{EE} / \mathrm{ED} \mathrm{s} / \mathrm{VCT}$ & $\begin{array}{l}8 \mathrm{sem} \\
3 \mathrm{x} \mathrm{sem}\end{array}$ & $\begin{array}{c}\text { Dinamómetro } \\
\text { Quadril (F/E) } \\
\text { Joelho (F/E) } \\
\text { Tornozelo } \\
\text { (FP/DF) } \\
\text { Pré / Pós }\end{array}$ & $\begin{array}{c}\text { Quadril: ND } \\
\text { Joelho: VCT: F } \\
\uparrow^{*} 12.3 ; \mathrm{E}: \uparrow^{*} 10.6 \% \\
\text { CON: } \uparrow^{*} 6.3 \% ; \\
\text { E: } \uparrow^{*} 7.9 \% \\
\text { Tornozelo: VCT: } \\
\text { DF:ND; FP } \uparrow^{*}{ }^{\mathrm{F}} 20.4 \% \\
\text { CON: DF:ND; FP: } \\
\text { ND }\end{array}$ & $5 / 10$ \\
\hline
\end{tabular}

Nota: A - Ativos; CAM- caminhada; CON- controle; DF - dorsiflexão; E- extensores; ED - exercício dinâmico; EE - exercício estático; F - Flexão; FP- flexão plantar; H - Homens;

I - Institucionalizados; J- joelho; M - mulheres; NR - não reportado; NS- não significativo; S - segundos; Sem - semanas; * diferença significativa quando comparada com os valores préintervenção; ${ }^{\mp}$ diferença significativa quando comparada com os valores do grupo controle; ${ }^{++}$diferença significativa quando comparada com os valores do grupo de CAM 
al., 2004, 2011; Von Stengel et al., 2011, 2012). As medidas de potência muscular foram realizadas a partir dos testes de salto (Raimundo et al., 2009; Von Stengel et al., 2011, 2012) e diante do dinamómetro isocinético (Da Silva et al., 2009; Machado et al., 2010; Rees et al., 2008). Um resumo de todos os estudos e seus desfechos está disponível nas tabelas 1 e 2 .

Dentre os 16 estudos encontrados, $81.3 \%$ apresentavam os resultados da análise metodológica disponíveis para consulta na base Pedro. Dos estudos que não apresentavam a análise da qualidade metodológica, dois foram identificados com baixa qualidade. Dos artigos que compõem a presente revisão, $68.8 \%$ apresentaram escores $\geq 5$ na escala de avaliação, considerados assim, de moderada a alta qualidade.

\section{DISCUSSÃO}

\section{Efeitos da VCT sobre a força muscular}

De modo geral, os estudos evidenciam o aumento da força muscular isométrica e dinâmica diante da intervenção com VCT, incluindo programas, variando entre 2 e 18 meses. Apenas três estudos não identificaram efeito induzido pela VCT sobre a força muscular (Bautmans et al., 2005; Da Silva et al., 2009; Raimundo et al., 2009).

Bautmans et al. (2005) não observaram modificações na força muscular dos extensores do joelho de mulheres idosas institucionalizadas, após 6 semanas de intervenção com VCT (35-40Hz; $2-5 \mathrm{~mm})$. No entanto, mesmo sem efeitos sobre a variável de desempenho muscular, observou-se uma melhoria significativa nas tarefas funcionais no grupo de vibração, quando comparadas à condição controle. A falta de descrição do ângulo de flexão durante a isometria e o facto de os autores avaliarem a força de forma dinâmica, apesar dos sujeitos terem sofrido a intervenção com exercícios estáticos, limitam a interpretação destes achados. Além disto, ressalta-se a curta duração do estudo (6 semanas), e o baixo tempo total do estímulo vibratório (3 a 6 minutos), quando comparado a outros estudos (Tabela 1). De forma similar, Raimundo et al. (2009) também não observa- ram efeitos dos exercícios isométricos concomitantes a VCT $(12.6 \mathrm{~Hz} ; 3-6 \mathrm{~mm})$ sobre a força muscular dinâmica de mulheres, após 8 meses de intervenção. Entretanto, a amostra reduzida, a utilização de uma baixa frequência de VCT $(12.6 \mathrm{~Hz})$, assim como a falta de informações sobre a aderência ao programa, representam importantes ameaças à validade interna deste experimento.

Da Silva et al. (2009) também não identificaram a presença de efeitos nas variáveis de força e potência muscular, após 13 semanas de exercícios isométricos com VCT $(30-40 \mathrm{~Hz}$; 2.0-6.0mm) em idosos ativos. No entanto, a presença de limitações metodológicas restringe a interpretação destes resultados, como a ausência de randomização, falta de uma condição controle para as posturas utilizadas durante a VCT, ausência de critérios de elegibilidade para as amostras, variação entre os géneros e a carência dos dados de aderência ao programa.

Além das limitações metodológicas apresentadas, é possível identificar na Tabela 1, que os estudos que não apontaram efeito da intervenção com VCT sobre a força muscular contemplam a realização exclusiva de exercícios isométricos e em contrapartida, a mensuração da variável resposta envolve medidas de produção de força dinâmica (Bautmans et al., 2005; Da Silva et al., 2009; Raimundo et al., 2009). Tais condições podem justificar os resultados encontrados.

Embora a maior parte da literatura revisada assinale uma melhora significativa da força muscular a partir da VCT (Tabela 1), e também apresente indicativos de alta a moderada qualidade metodológica, a presença de algumas variáveis intervenientes merecem ser consideradas para um melhor esclarecimento dos resultados encontrados.

Bogaerts et al. (2011) e Machado et al. (2010) identificaram um aumento significativo da força dos extensores de joelho de idosos, após 6 meses (força isométrica - 14.9\%) e 10 semanas (força isométrica $-38 \%$ ), respetivamente, de exercícios estáticos e dinâmicos associados a VCT, 1.6-2.2g/30-40 Hz (Bogaerts 
et al., 2011) e $2.0-4.0 \mathrm{~mm} / 20-40 \mathrm{~Hz}$ (Machado et al., 2010), quando comparados com o grupo controle. Após 6 meses de intervenção com VCT foi identificada uma menor adaptação muscular quando comparado com a rotina de apenas 10 semanas de intervenção com vibração. Tais resultados podem ser explicados pelo facto da amostra ser composta por idosos institucionalizados.

Após 12 meses de um programa de exercícios dinâmicos associados à VCT do tipo vertical $(35 \mathrm{~Hz} ; 1.7 \mathrm{~mm})$ mulheres pós-menopausa apresentaram aumento de $24.4 \%$ na força dos extensores de joelho e na densidade mineral óssea da lombar. Os mesmos efeitos foram observados no grupo de idosas que foram submetidas à vibração através de uma plataforma do tipo rotatória $(12.5 \mathrm{~Hz} ; 12 \mathrm{~mm})$ quando comparado com o grupo controle (Von Stengel et al., 2011).

Analisando os estudos que utilizaram um desenho experimental com situação controle do estímulo vibratório (Bautmans et al., 2005; Mikhael et al., 2010; Rees et al., 2007, 2008; Von Stengel et al., 2012), observa-se que a maior parte dos estudos não demonstrou efeitos adicionais diante da VCT. Portanto, podese atribuir que as modificações parecem resultar das adaptações musculares provocadas pelos exercícios e não pelo estímulo vibratório.

Em contrapartida, efeitos adicionais foram encontrados em dois estudos conduzidos pelo mesmo grupo de pesquisadores (Rees et al., 2007, 2008), com desenhos experimentais, número e característica dos participantes muito semelhantes, incluindo grupos independentes, onde um foi submetido a oito semanas com uma frequência de três sessões semanais de VCT $(26 \mathrm{~Hz} ; 5-8 \mathrm{~mm})$ associados a exercícios estáticos e dinâmicos (EX+VCT), enquanto um outro grupo foi conduzido a uma condição controle (EX), incluindo exatamente os mesmos procedimentos, exceto o estímulo de vibração. No primeiro, Rees et al. (2007) não identificaram efeitos sobre a força muscular do quadril nos grupos investigados, no entanto, o grupo de EX+VIB (8.1\%) e o EX (7.2\%) apre- sentaram valores similares para o aumento da força dinâmica dos extensores do joelho quando comparados com os valores pré-intervenção. Apenas os resultados para o desempenho muscular dos flexores plantares apresentaram um aumento significativo (18.5\%) no grupo EX+VCT. No estudo subsequente os autores (Rees et al., 2008) observaram resultados similares, onde os valores de força muscular dinâmica dos flexores plantares só apresentaram diferença significativa no grupo de vibração (18.2\%), enquanto nenhuma mudança foi identificada no grupo EX. Diante da condição controle apresentada nos dois ensaios experimentais (Rees et al., 2007, 2008), parece que a VCT foi capaz de induzir maiores adaptações na resposta muscular do tríceps sural do que apenas a partir dos exercícios dinâmicos e estáticos. Entretanto, os mesmos efeitos não foram observados no desempenho muscular dos flexores e extensores do quadril e do joelho.

A divergência na caracterização da amostra, as diferentes formas de mensuração da variável resposta, a grande heterogeneidade dos protocolos de vibração, contendo as variáveis de volume e intensidade, e a ampla variação nos desenhos experimentais (Tabela 1), prejudicam a comparação e inferência de tais resultados. Nos estudos revisados é evidente a constante falta de controle dos procedimentos realizados associados ao estímulo vibratório, sendo que a principal limitação consiste na ausência de um grupo controle incluindo os mesmos exercícios realizados no grupo de VCT (Bemben et al., 2010; Bogaerts et al., 2007, 2009, 2011; Da Silva et al., 2009; Machado et al., 2010; Roelants et al., 2004; Verschueren et al., 2011; Von Stengel et al., 2011). Tal limitação torna os achados questionáveis, já que não é possível identificar se os resultados são realmente induzidos pela vibração.

\section{Efeitos dos exercícios concomitantes à VCT versus outras modalidades de intervenção}

Bogaerts et al. (2007, 2009) identificaram em dois estudos com o mesmo procedimento experimental, que os modelos de intervenção 
incluindo a VCT concomitantes a exercícios estáticos e dinâmicos versus os exercícios incluindo multimodalidades - alongamento, aeróbio, coordenação, força e equilíbrio - apresentaram efeitos similares no desempenho muscular isométrico dos extensores do joelho, variando de 9.4 a $9.8 \%$ no grupo de VCT e de 9.8 a $13.1 \%$ no grupo de multimodalidades.

Os estudos que compararam as condições experimentais de VCT versus o ER apresentaram achados contraditórios (Bemben et al., 2010; Roelants et al., 2004). Roelants et al. (2004) verificaram um aumento significativo na força muscular isométrica $(15.0 \%)$ e dinâmica $(16.1 \%)$ induzido a partir de 24 semanas de intervenção incluindo exercícios estáticos e dinâmicos associados à VCT $(35-40 \mathrm{~Hz}$; 2.5 $5.5 \mathrm{~mm})$. No entanto, efeitos similares ( $18.4 \%$ na força isométrica e $13.9 \%$ na força dinâmica) foram encontrados no grupo submetido ao ER (cadeira extensora e Leg-Press - 2x, 12 a 15 RM). Em um ensaio clínico controlado e randomizado, com duração de 24 semanas, Verschueren et al. (2004) encontraram achados similares: um aumento significativo de $15 \%$ e de $16.5 \%$ foi apresentado no grupo de VCT para as variáveis de força muscular isométrica e dinâmica dos extensores do joelho, respectivamente. Os resultados para o desempenho muscular dos extensores do joelho, no grupo de idosos submetidos a ER, também demonstraram aumento significativo (variando de 10.6 a $16 \%$ ), sem no entanto, diferença significativa entre os grupos experimentais.

Contrariando estes achados, Bemben et al. (2010) evidenciaram um aumento significativo de $60 \%$ na força muscular dos abdutores, assim como de $68 \%$ na adução do quadril, após 8 meses de intervenção de VCT (2-4 mm; 30$40 \mathrm{~Hz}$ ), associada ao ER (3x 10 rep; 80\% 1RM), quando comparado aos efeitos do ER isolado. Entretanto, nos dois grupos foram encontrados efeitos similares para força muscular dos extensores/flexores do quadril e extensores do joelho. Uma possível justificativa, para tais resultados, pode ser o padrão em cadeia cinética fechada realizada a partir da intervenção com VCT. Tais efeitos podem ser atribuídos à especificidade da posição em agachamento, onde há uma demanda constante de ativação dos músculos adutores e abdutores que atuam como importantes estabilizadores pélvicos. Desta maneira, considerando a especificidade e a falta de um grupo controle incluindo apenas a posição em cadeia cinética fechada sem o estímulo vibratório, questiona-se o maior desempenho muscular dos abdutores e adutores do quadril a partir do estímulo mecânico de vibração.

Resumindo, a ausência de uma condição controle, com os mesmos exercícios usados e posturas semelhantes às da VCT, sugerem um fator de confundimento na interpretação dos resultados.

\section{Efeitos da VCT sobre a potência muscular}

A manifestação rápida da força de membros inferiores tem sido sugerida como um melhor preditor de quedas e de desempenho funcional de idosos (Izquierdo, Aguado, Gonzalez, Lopez, \& Hakkinen, 1999). Estudos têm verificado que idosos com histórico de quedas demonstram menor potência nos músculos da perna do que idosos não caidores (Perry, Carville, Smith, Rutherford, \& Newham, 2007; Skelton, Kennedy, \& Rutherford, 2002). Dentro deste contexto, torna-se relevante verificar se os efeitos da VCT estão associados ao aumento da potência muscular.

Machado et al. (2010) não identificaram efeito sobre a potência muscular dos extensores do joelho, após 10 semanas de intervenção de exercícios dinâmicos e estáticos associados à VCT (2.0-4.0mm; 20-40Hz). Da mesma forma, outros autores (Bautmans et al., 2005; Da Silva et al., 2009; Mikhael et al., 2010; Von Stengel et al., 2011) também não observaram efeitos induzidos da VCT sobre a potência muscular, após programas de 6 semanas a 12 meses de intervenção.

Em contrapartida, Rees et al. (2008) observaram um aumento de $12.3 \%$ na potência muscular dos flexores e de $10.6 \%$ dos extensores do joelho, a partir de 8 semanas de exercí- 
cios estáticos e dinâmicos associados à VCT. O grupo controle, que foi submetido às mesmas intervenções da condição experimental, exceto pela adição do estímulo vibratório, apresentou aumentos similares da potência muscular dos flexores e extensores do joelho, variando de $6.3 \%$ a $7.9 \%$. Os benefícios superiores da VCT, quando comparados com a condição controle, só foram evidenciados para a potência muscular dos flexores plantares, que apresentaram ganhos de $20.4 \%$ após a intervenção. Nenhum efeito adicional foi encontrado para a potência muscular dos flexores e extensores do quadril e para os músculos dorsiflexores do tornozelo. Considerando que o controle do equilíbrio corporal também é promovido a partir das estratégias motoras do tornozelo, do quadril e do passo, os ganhos evidenciados na potência dos flexores plantares após o estímulo vibratório podem contribuir de alguma forma para a melhora na manutenção da estabilidade corporal e dos ajustes antecipatórios, entretanto, como isso não foi investigado no estudo citado a possível inferência desses achados é apenas uma especulação. Raimundo et al. (2009) também encontraram um aumento de $7 \%$ na potência muscular, a partir do salto vertical, após 8 meses de intervenção, mesmo diante de uma baixa frequência de VCT $(12.6 \mathrm{~Hz})$, quando comparado com o grupo que realizou apenas exercícios aeróbios.

A quantidade limitada de estudos e os achados divergentes na literatura considerando a influência da VCT sobre a potência muscular de idosos restringe fortemente a interpretação destes achados.

\section{Prescrição da VCT na intervenção terapêutica de idosos}

Doze estudos (75\% dos trabalhos) propõem um programa com uma frequência de três sessões semanais, intercaladas por um dia, sendo utilizado em $43.7 \%$ dos estudos a realização de um programa com uma frequência de 30 a 40 $\mathrm{Hz}$. O volume típico encontrado, considerando a duração do estímulo de VCT, incluiu protocolos de 30 a $60 \mathrm{~s}$ aplicados concomitantes aos exercícios estáticos e dinâmicos e a realização de 1 a 3 séries com $60 \mathrm{~s}$ de repouso.

Contrariando a maior parte dos desenhos experimentais conduzidos, Mikhael et al. (2010) utilizaram um estímulo intermitente de 20 minutos de vibração (1 minuto de estímulo por 1 minuto de repouso) e observaram, após 13 semanas $(12 \mathrm{~Hz} ; 1 \mathrm{~mm})$, aumento significativo da força muscular, mensurado a partir do agachamento (1RM - 10.7 a $14.4 \%$ ).

Na prática clínica é comum a utilização da VCT, incluindo protocolos de agachamento associando as atividades isométricas, concêntricas e excêntricas. As evidências disponíveis apontam para a manutenção destas rotinas, uma vez que os estudos que conduziram o programa de intervenção apenas com exercícios estáticos não obtiveram efeitos sobre a força muscular de idosos (Bautmans et al., 2005; Da Silva et al., 2009; Raimundo et al., 2009). No entanto, poucos estudos contemplam a informação da amplitude de movimento do joelho durante o estímulo com VCT (Mikhael et al., 2010). Desta forma, torna-se necessário o desenvolvimento de novos ensaios clínicos objetivando verificar os efeitos de diferentes amplitudes de movimento do joelho e suas implicações sobre o desempenho muscular em indivíduos idosos.

\section{Mecanismos associados ao efeito do estímulo de vibração sobre a força muscular}

A hipótese defendida na literatura da especialidade é que diante da ação mecânica da vibração alterações no comprimento da unidade músculo-tendão são produzidas e este estímulo é detetado pelos recetores sensoriais desencadeando uma resposta do sistema neuromuscular de contração muscular reflexa (Cardinale \& Bosco, 2003), conhecida como reflexo vibratório tónico (RVT) (Abercromby et al., 2007; Hazell, Jakobi, \& Kenno, 2007; Roelants, Verschueren, Delecluse, Levin, \& Stijnen, 2006). A onda vibratória é considerada um poderoso estímulo dos aferentes Ia do fuso muscular (Roll \& Vedel, 1982; Roll, Vedel, \& Ribot, 1989). 
A melhora na coordenação intra e intermuscular induzida pelo aumento temporário do drive neural para os músculos com um maior recrutamento e sincronização das unidades motoras provocadas durante a aplicação do estímulo vibratório são defendidos como possíveis mecanismos que colaboram para $\mathrm{o}$ aumento do controle neuromuscular (Abercromby et al., 2007; Hazell et al., 2007; Roelants et al., 2006). Entretanto, tais mecanismos carecem de evidência experimental.

As modificações no trofismo muscular são apresentadas como os mecanismos estruturais envolvidos na otimização da força muscular, a partir da VCT. Machado et al. (2010) identificaram um aumento variando de 8.7 a $15.5 \%$ na área de secção transversa, medida a partir de tomografia computadorizada, para o bíceps femoral e o vasto medial após 10 semanas de intervenção de exercícios estáticos e dinâmicos associados à VCT $(20-40 \mathrm{~Hz} ; 2-4 \mathrm{~mm})$. De forma similar, Bogaerts et al. (2007) relataram um aumento significativo de $3.4 \%$ no volume de massa muscular $\left(\mathrm{cm}^{3}\right)$ da perna - análise através da tomografia computadorizada - após 12 meses de intervenção com VCT $(30-40 \mathrm{~Hz}$; 2.5-5.0mm).

No entanto, Mikhael et al. (2010) e Verschueren et al. (2011) não identificaram a presença de tais modificações após a VCT, incluindo um programa de intervenção de 13 semanas e 6 meses, respetivamente. No estudo conduzido por Mikhael et al. (2010) cabe ressaltar que a VCT de baixa frequência $(12 \mathrm{~Hz}), \mathrm{o}$ ângulo de $20^{\circ}$ de flexão do joelho e a característica estática do exercício, podem ter contribuído para a ausência de efeitos após a intervenção. Outra limitação consiste na reduzida amostra de investigação.

\section{CONCLUSÕES}

Os resultados encontrados, limitados pelos procedimentos experimentais utilizados, sugerem que a VCT concomitante aos exercícios isométricos e dinâmicos promovem aumento da força muscular de idosos. No entanto, nos experimentos com a presença da condição con- trole dos exercícios utilizados durante o estímulo vibratório, observa-se que a maior parte dos estudos não demonstra efeitos adicionais decorrentes da VCT. Estes achados sugerem que as adaptações musculares possivelmente podem ter sido provocadas pelos exercícios e não necessariamente pelo estímulo vibratório empregado, pelo menos nas frequências de vibração utilizadas (12 a $40 \mathrm{~Hz}$ ).

Diante da quantidade limitada (31.2\%) de estudos que se preocuparam em incluir um grupo controle constituído dos mesmos exercícios realizados no grupo de vibração, assim como diante da grande diversidade dos protocolos empregados nas rotinas de vibração, ainda não é possível determinar se o estímulo vibratório, por si só, é capaz de promover efeitos sobre a força muscular de idosos. A partir dos experimentos realizados, não foi identificada uma relação de dose-resposta do estímulo vibratório, variável fundamental na tentativa de nortear a prática clínica na população alvo. A quantidade limitada de estudos e os achados divergentes na literatura destacam a necessidade de cautela na inferência dos dados sobre o efeito da VCT sobre a potência muscular.

Além disso, poucos estudos $(43.7 \%)$ comparam a VCT com outros métodos de intervenção para a população idosa, e mesmo considerando as limitações dos diferentes métodos adotados (exercício resistido, aeróbios e de multimodalidades), os resultados, até ao momento, não indicaram efeitos adicionais a partir do estímulo vibratório quando comparados com outras intervenções.

Desta forma, torna-se relevante o desenvolvimento de novos ensaios clínicos controlados e randomizados na tentativa de identificar a influência de diferentes magnitudes de estímulos vibratórios (diferentes frequências e durações de estímulo vibratórios, empregados com diferentes intervalos ente estímulo e recuperação e diferentes volumes de treino) e o consequente efeito induzido no desempenho muscular. A comparação dos diferentes métodos de treino com a vibração também necessita de ser melhor estudada. 


\section{Agradecimentos: \\ Nada a declarar.}

Conflito de Interesses:

Nada a declarar.

\section{Financiamento:}

M.O. Campos é bolsista do curso de doutoramento em Ciências do Exercício e do Esporte da Universidade Gama Filho, Rio de Janeiro, Brasil. P.S.C. Gomes é bolsista de Produtividade do CNPq, Brasil.

\section{REFERÊNCIAS}

Abercromby, A. F., Amonette, W. E., Layne, C. S., McFarlin, B. K., Hinman, M. R., \& Paloski, W. H. (2007). Variation in neuromuscular responses during acute whole-body vibration exercise. Medicine $\mathcal{E}$ Science in Sports $\mathcal{E}$ Exercise, 39(9), 1642-1650. doi: 10.1249/mss.0b013e31 $8093 \mathrm{f} 551$

Baumgartner, R. N., Koehler, K. M., Gallagher, D., Romero, L., Heymsfield, S., Ross, R. R., ... Lindeman, R. (1998). Epidemiology of sarcopenia among the elderly in New Mexico. American Journal of Epidemiology, 147(8), 755-763.

Bautmans, I., Van Hees, E., Lemper, J. C., \& Mets, T. (2005). The feasibility of whole body vibration in institutionalized elderly persons and its influence on muscle performance, balance and mobility: A randomised controlled trial. BMC Geriatrics, 5, 17. doi: 10.1186/1471-2318-5-17

Bemben, D. A., Palmer, I. J., Bemben, M. G., \& Knehans, A. W. (2010). Effects of combined whole-body vibration and resistance training on muscular strength and bone metabolism in postmenopausal women. Bone, 47(3), 650-656. doi: 10.1016/j.bone.2010.06.019

Bogaerts, A., Delecluse, C., Claessens, A., Coudyzer, W., Boonen, S., \& Verschueren, S. M. (2007). Impact of whole-body vibration training versus fitness training on muscle strength and muscle mass in older men: A 1-year randomized controlled trial. The Journal of Gerontology: Biological Sciences, 62(6), 630-635.

Bogaerts, A., Delecluse, C., Claessens, A. L., Troosters, T., Boonen, S., \& Verschueren, S. M. (2009). Effects of whole body vibration training on cardiorespiratory fitness and muscle strength in older individuals (a 1-year randomised controlled trial). Age and Ageing, 38(4), 448-454. doi: 10.1093/ageing/afp067

Bogaerts, A., Delecluse, C., Boonen, S., Claessens, A. L., Milisen, K., \& Verschueren, S. M. (2011). Changes in balance, functional performance and fall risk following whole body vibration training and vitamin D supplementation in institutionalized elderly women: A 6 month randomized controlled trial. Gait Posture, 33(3), 466-472. doi: 10.1016/j.gaitpost.2010.12.027

Cardinale, M., \& Bosco, C. (2003). The use of vibration as an exercise intervention. Exercise and Sport Sciences Reviews, 31, 3-7.

Chu, L. W., Chiu, A. Y., \& Chi, I. (2006). Impact of falls on the balance, gait, and activities of daily living functioning in community-dwelling Chinese older adults. The Journal of Gerontology Biological Sciences and Medical Sciences, 61(4), 399404.

Da Silva, R., Andreotti, R., Gehring, P., Nunes, M., Wallerstein, L., Fonseca, M., ... Ugrinowtsch, C. (2009). Efeito do treinamento vibratório na força muscular e em testes funcionais em idosos fisicamente ativos. Revista Brasileira de Cineantropometria e Desempenho Humano, 11(2), 166-173.

Di Monaco, M., Vallero, F., Di Monaco, R., \& Tappero, R. (2010). Prevalence of sarcopenia and its association with osteoporosis in 313 older women following a hip fracture. Archives of Gerontology and Geriatrics, 52(1), 71-74. doi: 10.1016/j.archger.2010.02.002

Erim, Z., Beg, M. F., Burke, D. T., \& de Luca, C. J. (1999). Effects of aging on motor-unit control properties. Journal of Neurophysiology, 82(5), 2081-2091.

Evans, J. R., Fletcher, A. E., Wormald, R. P., Ng, E. S., Stirling, S., Smeeth, L., ... Tulloch, A. (2002). Prevalence of visual impairment in people aged 75 years and older in Britain: Results from the MRC trial of assessment and management of older people in the community. British Journal of Ophthalmology, 86(7), 795-800.

Fathi, D., Ueki, Y., Mima, T., Koganemaru, S., Nagamine, T., Tawfik, A., \& Fukuyama, H. (2010). Effects of aging on the human motor cortical plasticity studied by paired associative stimulation. Clinical Neuroembryology, 121(1), 90-93. doi: 10.1016/j.clinph.2009.07.048

Frontera, W. R., Hughes, V. A., Fielding, R. A., Fiatarone, M. A., Evans, W. J., \& Roubenoff, R. 
(2000). Aging of skeletal muscle: A 12-yr longitudinal study. Journal of Applied Physiology, 88(4), 1321-1326.

Hakkinen, K., Kraemer, W. J., Newton, R. U., \& Alen, M. (2001). Changes in electromyographic activity, muscle fibre and force production characteristics during heavy resistance/power strength training in middle-aged and older men and women. Acta Physiologica Scandinavica, 171 (1), 51-62. doi: 10.1046/j.1365-201X.2001. 00781

Hazell, T. J., Jakobi, J. M., \& Kenno, K. A. (2007). The effects of whole-body vibration on upperand lower-body EMG during static and dynamic contractions. Applied Physiology, Nutrition, and Metabolism, 32(6), 1156-1163. doi: 10.1139/ h07-116

Izquierdo, M., Aguado, X., Gonzalez, R., Lopez, J. L., \& Hakkinen, K. (1999). Maximal and explosive force production capacity and balance performance in men of different ages. European Journal of Applied Physiology, 79(3), 260-267.

Kent-Braun, J. A., Ng, A. V., \& Young, K. (2000). Skeletal muscle contractile and noncontractile components in young and older women and men. Journal of Applied Physiology, 88, 662-668.

Kubo, K., Kanehisa, H., Miyatani, M., Tachi, M., \& Fukunaga, T. (2003). Effect of low-load resistance training on the tendon properties in middle-aged and elderly women. Acta Physiologica Scandinavica, 178(1), 25-32. doi: 10.1046/j.1365-201X.2003.01097

Machado, A., Garcia-Lopez, D., Gonzalez-Gallego, J., \& Garatachea, N. (2010). Whole-body vibration training increases muscle strength and mass in older women: A randomized-controlled trial. Scandinavian Journal of Medicine \& Science in Sports, 20(2), 200-207. doi: 10.1111/j.16000838.2009.00919.

Mikhael, M., Orr, R., Amsen, F., Greene, D., \& Singh, M. A. (2010). Effect of standing posture during whole body vibration training on muscle morphology and function in older adults: A randomised controlled trial. BMC Geriatrics, 10, 74. doi: 10.1186/1471-2318-10-74

Miljkovic-Gacic, I., Gordon, C. L., Goodpaster, B. H., Bunker, C. H., Patrick, A. L., Kuller, L. H., ... Zmuda, J. M. (2008). Adipose tissue infiltration in skeletal muscle: Age patterns and association with diabetes among men of African ancestry. The American Journal of Clinical Nutrition, 87(6), 1590-1595.
Moseley, A. M., Herbert, R. D., Sherrington, C., \& Maher, C. G. (2002). Evidence for physiotherapy practice: A survey of the Physiotherapy Evidence Database (PEDro). Australian Journal of Physiotherapy, 48(1), 43-49.

Nelson, M. E., Layne, J. E., Bernstein, M. J., Nuernberger, A., Castaneda, C., Kaliton, D., ... Fiatarone Singh, M. A. (2004). The effects of multidimensional home-based exercise on functional performance in elderly people. The Journal of Gerontology Biological Sciences and Medical Sciences, 59(2), 154-160.

Onambele, G. L., Narici, M. V., \& Maganaris, C. N. (2006). Calf muscle-tendon properties and postural balance in old age. Journal of Applied Physiology, 100(6), 2048-2056.

Perry, M. C., Carville, S. F., Smith, I. C., Rutherford, O. M., \& Newham, D. J. (2007). Strength, power output and symmetry of leg muscles: Effect of age and history of falling. European Journal of Applied Physiology, 100(5), 553-561. doi: 10.1007/s00421-006-0247-0

Raimundo, A. M., Gusi, N., \& Tomas-Carus, P. (2009). Fitness efficacy of vibratory exercise compared to walking in postmenopausal women. European Journal of Applied Physiology, 106(5), 741-748. doi: 10.1007/s00421-009-1067-9

Rees, S. S., Murphy, A., \& Watsford, M. (2007). Effects of vibration exercise on muscle performance and mobility in an older population. Journal of Aging and Physical Activity, 15, 367-381.

Rees, S. S., Murphy, A. J., \& Watsford, M. L. (2008). Effects of whole-body vibration exercise on lower-extremity muscle strength and power in an older population: A randomized clinical trial. Physical Therapy, 88(4), 462-470. doi: 10.2522/ptj.20070027

Roelants, M., Delecluse, C., \& Verschueren, S. M. (2004). Whole-body-vibration training increases knee-extension strength and speed of movement in older women. Journal of the American Geriatrics Society, 52(6), 901-908. doi: 10.1111/j.1532-5415.2004.52256.

Roelants, M., Verschueren, S. M., Delecluse, C., Levin, O., \& Stijnen, V. (2006). Whole-bodyvibration-induced increase in leg muscle activity during different squat exercises. The Journal of Strength \& Conditioning Research, 20(1), 124-129.

Roll, J. P., \& Vedel, J. P. (1982). Kinaesthetic role of muscle afferents in man, studied by tendon vibration and microneurography. Experimental Brain Research, 47(2), 177-190. 
Roll, J. P., Vedel, J. P., \& Ribot, E. (1989). Alteration of proprioceptive messages induced by tendon vibration in man: A microneurographic study. Experimental Brain Research, 76(1), 213222.

Rooyackers, O. E., Adey, D. B., Ades, P. A., \& Nair, K. S. (1996). Effect of age on in vivo rates of mitochondrial protein synthesis in human skeletal muscle. Proceedings of the National Academy of Sciences of the United States of America, 93(26), 15364-15369.

Ryan, A. S., Ivey, F. M., Hurlbut, D. E., Martel, G. F., Lemmer, J. T., Sorkin, J. D., ... Hurley, B. F. (2004). Regional bone mineral density after resistive training in young and older men and women. Scandinavian Journal of Medicine $\mathcal{E}$ Science in Sports, 14(1), 16-23. doi: 10.1111/j.16000838.2003.00328

Short, K., Bigelow, M., Kahl, J., Singh, R., CoenenSchimke, J., Raghavakaimal, S., \& Nair, K. S. (2005). Decline in skeletal muscle mitochondrial function with aging in humans. Proceedings of the National Academy of Sciences of the United States of America, 102(15), 5618-5623. doi: 10.1073/pnas.0501559102

Skelton, D., Kennedy, J., \& Rutherford, O. (2002). Explosive power and asymmetry in leg muscle function in frequent fallers and non-fallers aged over 65. Age and Ageing, 31 (2), 119-125.
Verschueren, S., Bogaerts, A., Delecluse, C., Claessens, A., Haentjens, P., Vanderschueren, D., \& Boonen, S. (2011). The effects of whole-body vibration training and vitamin $\mathrm{D}$ supplementation on muscle strength, muscle mass, and bone density in institutionalized elderly women: A 6-month randomized, controlled trial. Journal of Bone and Mineral Research, 26(1), 42-49. doi: 10.1002/jbmr.181

Verschueren, S. M., Roelants, M., Delecluse, C., Swinnen, S., Vanderschueren, D., \& Boonen, S. (2004). Effect of 6-month whole body vibration training on hip density, muscle strength, and postural control in postmenopausal women: A randomized controlled pilot study. Journal of Bone and Mineral Research, 19(3), 352-359. doi: 10.1359/ jbmr.0301245

Von Stengel, S., Kemmler, W., Bebenek, M., Engelke, K., \& Kalender, W. A. (2011). Effects of whole-body vibration training on different devices on bone mineral density. Medicine $\mathcal{E}$ Science in Sports \& Exercise, 43(6), 1071-1079. doi: 10.1249/MSS.0b013e318202f3d3

Von Stengel, S., Kemmler, W., Engelke, K., \& Kalender, W. A. (2012). Effect of whole-body vibration on neuromuscular performance and body composition for females 65 years and older: A randomized-controlled trial. Scandinavian Journal of Medicine $\mathcal{E}$ Science in Sports, 22(1), 119-127. doi: 10.1111/j.1600-0838.2010.01126 